\title{
A Matched Case-Control Study of Beans Intake And Breast Cancer Risk in Urbanized Nigerian Women.
}

Galya Bigman ( $\sim$ bigman.galya@gmail.com )

University of Maryland School of Medicine https://orcid.org/0000-0002-4747-6888

\section{Sally Adebamowo}

University of Maryland School of Medicine

King-David Terna Yaw

University of Abuja Teaching Hospital

Monday Yilkudi

University of Abuja Teaching Hospital

Oluwole Olaomi

National Hospital Abuja

Olawale Badejo

National Hospital Abuja

Ayo Famooto

Institute of Human Virology

Emmanuel Ezeome

University of Nigeria Teaching Hospital

lliya Karmiliyus Salu

Asokoro District Hospital

Elijah Miner

Garki Hospital

Ikrchukwu Anosike

Wuse General hospital

Benjamin Achusi

Federal Staff Hospital

Clement Adebamowo

University of Maryland School of Medicine

\section{Research Article}

Keywords: Breast Cancer, Triple-Negative, Hormone Receptor-Positive, Beans Intake, African Women

Posted Date: December 16th, 2021 
DOI: https://doi.org/10.21203/rs.3.rs-1044631/v1

License: (c) (i) This work is licensed under a Creative Commons Attribution 4.0 International License. Read Full License

Version of Record: A version of this preprint was published at Current Developments in Nutrition on May 29th, 2020. See the published version at https://doi.org/10.1093/cdn/nzaa044_009. 


\section{Abstract}

Purpose. Beans intake has been associated with reduced risk of breast cancer (BRCA), however; only few studies considered molecular subtypes status and none in African women. Therefore, the purpose of this study was to examine the associations between dietary intake of beans and BRCA including its subtypes in Nigerian women.

Methods. Overall, 472 newly diagnosed patients with primary invasive BRCA were age-matched $( \pm 5$ years) with 472 controls from the Nigerian Integrative Epidemiology of Breast Cancer (NIBBLE) Study from 01/2014-07/2016. We collected dietary intake of beans using a food frequency questionnaire (FFQ). Beans intake was categorized into three levels of never (never in the past year), low ( $\leq 1$ portion/week) and high intake ( $>1$ portions/week). We used conditional and unconditional logistic regression models to estimate the Odds Ratio (OR) of beans intake and the risk of overall BRCA and by its molecular subtypes.

Results. The mean (SD) age of cases was 44.4(10.0) and of controls was 43.5(9.5) years. In the case group, more than half $(51.1 \%)$ has never consumed beans alone in the past year compared to $39.0 \%$ in the control group. In multivariable models, we found significant inverse associations between beans intake and overall BRCA risk (OR=0.57; 95\%Cl: 0.38-0.85), hormone receptor-positive BRCA (OR=0.45, 95\% Cl: $0.23-0.90)$ and triple-negative BRCA (OR=0.47 95\%Cl: 0.25-0.88).

Conclusion. Dietary intake of beans of more than one portions a week is associated with reduced risk of BRCA in African women and it may play a significant role in reducing the incidence of BRCA particularly of the more aggressive triple-negative subtype, which is more prevalent in SSA.

\section{Introduction}

Breast cancer is the leading cause of cancer morbidity and mortality among women worldwide. Approximately one in four (24.2\%) of all new cancer cases diagnosed in women are breast cancer [1]. Although its incidence in low- and middle-income countries (LMIC) including Sub-Saharan Africa [2] is lower than that in high-income countries (HIC), the incidence in SSA is rising rapidly and it is now the commonest female cancer in Nigeria [2, 3]. From 2000 to 2015, the incidence of breast cancer in SSA increased from 21.9 (95\% Cl: 15.4-28.3) per 100,000 to 47.9 (95\% Cl: 41.2-54.6) per 100,000 [4].

The increase in the incidence of breast cancer in SSA can be explained by several risk factors including increased life expectancy, changes in obstetrics practices such as delays in commencement of childbearing, contraceptive use, reduced parity and reduced duration of breastfeeding $[5,6]$. Changes in lifestyle behaviors are also occurring in SSA due to rapid urbanization and socio-economic development leading to higher alcohol intake, and higher prevalence of obesity and physical inactivity, which all contribute to the overall increased cases in breast cancer in this area $[3,7,8]$.

Furthermore, SSA is undergoing nutritional transition particular among urbanized Africans. More urbanized Africans are departing from their traditional African diet rich in high fiber and plant-based 
proteins (e.g. beans) to consume more a 'Western diet' marked by high intake of meats, energy-dense and low fiber foods, as well as more intake of soft sweetened beverages $[9,10]$. Previous studies suggest that there are nutrient components in the traditional African diet that are associated with reduced risks of some cancer $[9,11]$. While, other components of the African diets, including certain foods and additives, the methods of foods storage and preparation may be associated with increased risk of other cancers [5]. On balance, departures from the traditional African diet as a result of the ongoing nutritional transition may contribute more to the increased incidence of breast cancer in African women but this remains largely unexplored [10].

Previous studies in other populations show that higher dietary intake of beans and legumes are associated with reduced risk of breast cancer [12-15]. In the Four-Corners Breast Cancer Case-Control Study, there was inverse association between breast cancer risk and factor-derived "Native Mexican" dietary pattern that loaded heavily with beans [13]. There have been few prospective studies examining the association between beans intake and breast cancer risk $[12,15]$. Adebamowo et. al. found inverse association between intake of beans and lentils, and breast cancer risk in Western women [12] and Velie et al. found that a factor-derived "traditional southern pattern" rich in legumes was inversely associated with breast cancer [15].

With the rising incidence of breast cancer in SSA along with the ongoing nutritional transitions, there is a unique opportunity to examine whether there is a link between these two changes. Therefore, the primary study aim was to examine the association between dietary intake of beans and breast cancer in Nigerian women. The secondary aim was to examine the association between dietary intake of beans and breast cancer subtypes (e.g., hormone receptor-positive, triple-negative) in Nigerian women, in particular the more aggressive subtype, triple-negative, which is more prevalent in SSA. Our hypothesis is that there will be a significant inverse association between high intake of dietary beans and lower risks of breast cancer including its subtypes in Nigerian women.

\section{Methods}

\section{Study design and setting}

We studied women enrolled in the Nigerian Integrative Epidemiology of Breast Cancer (NIBBLE) Study, a case-control study of female breast cancer that recruited participants at six government hospitals in Nigeria, five of whom are located in Abuja (National Hospital, University of Abuja Teaching Hospital Gwagwalada, Asokoro District Hospital, Garki Hospital and Wuse General Hospital) and the sixth hospital, the University of Nigeria Teaching Hospital, in Enugu, between January 2014 and July 2016. The details about the study design and setting have been previously published [16].

\section{Participants}

Overall, 508 newly diagnosed patients with primary invasive breast cancer aged 18 years or older were identified at their first visit to the clinical sites. Research nurses informed potential participants about the 
study and obtained their informed consent. Age-matched hospital-based controls (892) were women who did not have cancer or endocrine diseases and were within 5 years of the age of specific breast cancer patients enrolled within one month in the same hospital. Most (94.0\%) of the women approached consented to participate. Research nurses conducted face-to-face interviews in English language (70.6\%) or local Nigerian language (29.4\%) according to the patient's preference.

\section{Primary exposure}

Beans intake was calculated from the dietary intake data that were collected during face-to-face interview using a Semi-quantitative Food Frequency Questionnaire (FFQ) constructed from a list of 200 food items typically eaten in Nigeria. Participants indicated each food and beverage item they had eaten within the preceding year and the frequency with which they consumed the food (e.g. never, less than once a month, and up to several times a day) and the portion sizes they usually consumed (i.e. 1-serving spoon, 2serving spoons, 3-serving spoons). The FFQ asked about consumption of single items such as beans alone or common beans dishes such as porridge beans (Ewa Oloyin), stewed beans (Ewa Riro), beans soups (Adalu), beans pudding (Moin Moin), beans cakes (Akara). For the purposes of this study, we calculated intake of beans alone per week by multiplying the frequency of intake per week with reported portion size to create a continuous variable. Then, we categorized beans alone intake into three categories of never (never consumed beans in the past year), low ( $\leq 1$ portion per week) and high intake (>1 portions per week).

\section{Breast cancer and breast cancer subtypes}

Needle core biopsies were performed using Bard Magnum Biopsy Gun ${ }^{\circledR}$. Breast specimens were fixed in $10 \%$ neutral buffered formalin and processed within 48 hours of fixation with a minimum fixation time of 8 hours in Leica ${ }^{\circledR}$ automatic tissue processors at the African Collaborative Center for Microbiome and Genomics Research (ACCME) Laboratory at the Institute of Human Virology, Nigeria.

\section{Histology}

Sections of Paraffin-embedded blocks were cut at 3-4 $\mu \mathrm{m}$ and stained with hematoxylin and eosin stains. Histological features were classified according to the 2003 WHO classification of breast diseases and graded using the Nottingham modification of the Bloom-Richardson grading [17]. Only participants with final histologic confirmation of breast cancer were included in the analysis.

\section{Immunohistochemistry (IHC)}

Histologically confirmed invasive breast tumors were stained by immunohistochemical techniques using the Thermo Scientific Lab Vision primary antibodies (clones ER-SP1; PR-SP2; Her2-SP3) and Thermo Scientific ${ }^{\text {TM }}$ Ultra Vision ${ }^{\text {TM }}$ Quanto HRP DAB detection kit according to manufacturer's recommended protocol. In brief, formalin-fixed paraffin-embedded (FFPE) tissues were sectioned serially into $4 \mu \mathrm{m}$, placed in histogrip coated microscope slides, incubated overnight at $60 \mathrm{C}$, deparaffinized in series of xylene (three changes), graded alcohol (2 changes 100\%, 90\%, and $70 \%$ ethanol) and rehydrated in 
distilled water. Antigen retrieval was performed, sections were washed with Phosphate Buffered Saline (PBS) and blocked with hydrogen peroxide for 10 minutes. Then Ultra $V$ was applied to block nonspecific background staining for 5 minutes. Sections were washed with PBS and primary antibodies (ER-SP1; PRSP2; Her2-SP3) were applied on the sections and incubated at room temperature for 1 hour followed by application of primary antibody enhancer and HRP polymer. Staining was visualized using Diaminobenzidine (DAB) and counterstained with haematoxylin. Sections were dehydrated and cover slipped. We planned to perform immunohistochemistry (IHC) for all participants recruited into the NIBBLE study but in a few cases the core tissue biopsies were too small so IHC was not feasible.

\section{IHC interpretation}

We considered ER and PR were positive if $\geq 1 \%$ nuclei of the tumor cells were stained per the American Society College Oncology/College American Pathology (ASCO/CAP) guidelines [18]. HER2 staining was scored as $0,1+, 2+$, or $3+$ and we considered a staining of $3+$ (uniform, intense membrane staining of $>$ $30 \%$ of invasive tumor cells) a positive HER2 result [19].

\section{Breast cancer subtypes}

Overall, $57 \%$ of the cases $(292 / 508)$ had estrogen, progesterone and human epidermal growth factor 2 test results (Figure 1). We classified breast cancer subtypes using combinations of the IHC markers as follows (a) hormone receptor positive (HP) were tumors that had positive estrogen and progesterone tests but negative HER2 test and (b) triple-negative breast cancer (TNBC) were tumors that lacked all 3 markers [20]. Overall, 32.2\% ( $n=94 / 292)$ were classified as HP, and 42.1\% ( $n=123 / 292)$ as TNBC (Figure 2).

\section{Covariates}

We collected information on age in years, years of education completed (elementary, completed high school, post-high school with no university degree, completed university), marital status (married, single, separated/divorced/widowed), occupation (self employed, unskilled manual, skilled manual, professional executive), smoking experience (yes vs. no), alcohol use (yes vs. no), age at menarche, number of pregnancies which was categorized into $0,1-2,3-5,>5$ ), ever used oral contraceptive (yes vs. no), menopausal status (premenopausal vs. postmenopausal), and breastfeeding experience of more than one month (yes vs. no). The research nurses measured participants' height, weight, waist, and hip circumferences, and we derived body mass index (BMI $\mathrm{kg} / \mathrm{m}^{2}$ ) and waist-hip ratio (WHR) from these measurements. Participants with extreme values of WHR of less than 0.7 or higher than 1.6 or with a BMI less than $10 \mathrm{~kg} / \mathrm{m}^{2}$ or greater than $50 \mathrm{~kg} / \mathrm{m}^{2}$ were excluded from the analyses. BMI was categorized into $<25,25-29.9, \geq 30$ ) and WHR was categorized to $\leq 0.85$ and $>0.85$. To derive socio-economic status, we calculated the 'wealth index' using the following variables - house ownership and type of house owned (e.g. home, apartment, house or duplex); source of drinking water (e.g. from outside, well, borehole, piped or bottled); type of cooking fuel; use of separate room for cooking; type of toilet; and ownership of household goods including car and refrigerator. We used Principal Component Analysis (PCA) with varimax rotation to compute factor scores based on the sum of responses to these variables weighted by their factor loading. We used the first component in the PCA that explained $(35 \%)$ of the variations in the 
data, to generate a wealth index. The wealth index variable was used to classify participants to low socioeconomic status (lowest $40 \%$ of the score distribution), middle (middle $30 \%$ ) and high (highest 30\%) socio-economic class.

\section{Statistical Analysis \\ Overall breast cancer}

From the initial study sample $(n=1,400)$, we matched 472 cases with 472 controls based on age $( \pm 5$ year) using propensity score with the optimal matching procedure. Therefore, from the initial 508 cases, 36 were not matched due to age difference of 5 years or more and additional 420 unmatched controls were excluded making a final matched sample of 1:1 ratio with 944 participants (Figure 1). Compared to the final sample, excluded participants were significantly younger (37.8 \pm 9.7 vs. $44.0 \pm 9.7)(p$-value<0.001), and there were no significant differences in LTPA between excluded and included participants (data are not shown).

To examine bivariate associations between the independent variables by cases and controls, we implemented conditional logistic regressions for each independent variable separately. To construct our multivariable models, we selected independent variables with $p$-value $<0.20$ in the bivariate analysis for inclusion. Multiple imputations technique was performed to impute missing values of the independent variables after conducting missing completely at random test (MCAT) ( $p$-value $=0.63$ ). For multivariable analyses, we conducted conditional logistic regression models and used Wald tests to identify covariates with significant associations ( $p$-value $<0.05$ ) with risk of overall and molecular subtypes of breast cancer.

\section{Breast cancer subtypes}

We used unconditional logistic regression models to identify age-adjusted variables with $p$-value $<0.20$ in bivariate analyses. These variables were included in multivariable unconditional logistic regression models for each breast cancer subtype, separately.

We present the adjusted odds ratios (ORs) and their 95\% confidence intervals for breast cancer overall and by subtypes. Test of trend was conducted by treating the tertiles as ordered three-category variable. All analyses were performed using Stata SE version 15.1 (College Station, Texas) and R-Studio Version 1.1.447 - (c) 2009-2018 RStudio, Inc.

\section{Ethical approval}

Ethical approval was obtained from the National Health Research Ethics Committee of Nigeria, Health Research Ethics Committees in each participating hospital and the institutional ethics committee at the University of Maryland School of Medicine, Baltimore (US). The study was carried out in compliance with the Nigerian National Code for Health Research Ethics. All participants gave written informed consent in accordance with the Declaration of Helsinki and the Nigerian National Code for Health Research Ethics. 


\section{Results}

The characteristics of study sample are described in Table 1. The mean (SD) age between cases (44.4 (10.0)) was similar to controls (43.5 (9.5)) years after age matching. Cases and controls were similar in their level of socio-economic status, marital status, number of pregnancies, and breastfeeding experience. Women with breast cancer were older, had higher WHR and lower educational attainment, and were more likely to be post-menopausal and have non-professional jobs compared to women in the control group. Higher prevalence of obesity measured by $\mathrm{BMI} \geq 30$ was observed in controls vs. cases ( 31.2 vs. $39.2 \%$, pvalue $=0.01$ ) while higher abdominal fat measured by WHR was observed in cases compared to controls ( 69.9 vs. $64.8 \%$, p-value $=0.07)$. More than half of the cases $(51.1 \%)$ have never consumed beans alone in the past year compared to $39.0 \%$ in the controls. High intake of beans of more than one portion a week was higher in the controls (21.4\%) compared to cases (16.3\%).

Table 2 shows the final results from the multivariable conditional logistic regression models. After adjusting for associated covariates, there was a significant association between higher beans intake and lower odds of having breast cancer. Women who reported high level of beans intake had a significant reduction in their odds of having breast cancer compared to women who reported never consuming beans in the past year $(\mathrm{OR}=0.57,95 \% \mathrm{Cl}: 0.38-0.85$, $\mathrm{p}$-value=0.005) after adjusting for associated covariates.

Table 3 shows the final results of the multivariable logistic regression models examining beans intake and the risk of having breast cancer by its molecular subtypes. The adjusted model shows that women who had intake of more than one portion of beans in a week had significantly lower odds of having hormone receptor-positive breast cancer ( $\mathrm{OR}=0.45,95 \% \mathrm{Cl}$ : 0.23-0.90, $p$-trend=0.002) compared with those who reported never consuming beans in the past year. Similar results were observed in women with triple-negative breast cancer. Reduced odds of having triple-negative breast cancer was observed among those with high intake of beans compared with never (OR=0.47, 95\% Cl: $0.25-0.88, p$-trend $=0.001)$.

\section{Discussion}

Our study hypothesis was confirmed where we showed for the first time that dietary intake of beans is associated with reduced odds of overall, hormone receptor-positive and triple-negative breast cancer in African women after adjusting for well-established covariates including obesity, leisure time physical activity, menopausal status, marital status, number of pregnancies, breastfeeding experience, demographic variables, and type of occupation.

Our findings are similar to studies conducted in other populations [12-15]. For example, in prospective study of dietary beans intake, Adebamowo et. al. studied 90,630 nurses from the United States who participate in the Nurses' Health Study II, aged 26-46 years at baseline and followed for 8 years to examine their dietary intake and the association with breast cancer risk. The authors found that high cumulative average intake of beans or lentils, two or more times a week, compared with intake of less 
than once a month is associated with reduced risk of breast cancer (Relative Risk (RR) $=0.76,95 \% \mathrm{Cl}$ : 0.57-1.00; $p$-trend=0.03) [12]. Also, in experimental animal studies suggest inverse associations between consumption of a $60 \%$ bean diet with chemically induced breast cancer in rat models [23].

Other studies examined intake of beans while using factor analyses to derive dietary patterns. In a USwide prospective cohort study of 40,559 women by Velie et. al., three major dietary patterns were derived after using principal components factor analysis: vegetable-fish/poultry-fruit, beef/pork-starch, and traditional southern dietary patterns. The traditional southern pattern which is characterized by high intake of traditional rural southern US foods, including cooked greens, cooked beans and legumes, sweet potatoes, cornbread, cabbage, fried fish, chicken, and rice but low intake of cheese, mayonnaise-salad dressing, wine, liquor, and salty snacks was inversely associated with invasive breast cancer (Relative Hazard $(\mathrm{RH})=0.78,95 \% \mathrm{Cl}: 0.65-0.95 ; p$ trend $=0.003)[15]$.

In a case-control study of 2,135 breast cancer cases and 2,571 controls, the researchers showed that high intake of total beans was associated with reduced risk for breast cancer ( $\mathrm{OR}=0.81,95 \% \mathrm{Cl}$ : 0.66$1.01, p$-trend $=0.03$ ). There was an inverse association between estrogen and progesterone breast cancer and high intake of beans (OR=0.72, 95\% Cl: 0.50-1.05, $p$-trend= 0.04$)[14]$. In another case-control study of breast cancer in the United States, the Four-Corners Breast Cancer Study, Murtagh et. al. found an inverse association between breast cancer risk and factor-derived "Native Mexican" dietary pattern that heavily loaded with Mexican cheeses, soups, meat dishes, legumes and tomato-based sauces in Hispanic and non-Hispanic white women $(\mathrm{OR}=0.68,95 \% \mathrm{Cl}$ : 0.55-0.85; $p$-trend $<0.01)$ [13].

Beans is one of the commonest food items eaten in SSA and Latin America even though the amount consumed per capita is falling. In Nigeria, it is most commonly consumed alone or as porridge beans, stewed, soups, pudding or cakes. In this study, we examined intake of beans only because approximately half of our study participants consumed it in this form daily whereas consumption in other forms was less common. Other survey showed that approximately two third of Nigerians consume bean in different types of dishes daily [2]. Beans is an excellent source of plant proteins (23\%), complex carbohydrates, dietary fiber, flavonoids, some vitamins and minerals [24].

There are several mechanisms that might explain our study findings. The first one is the high fiber content in beans. Animal studies have shown that soluble fiber reduced mammary tumor growth, angiogenesis and metastasis in mice [25]. Fiber has been associated with slowing glucose absorption, reducing insulin secretion, and regulation of the bioavailability of insulin-like growth factors, which are important pathways in breast carcinogenesis $[25,26]$. Epidemiological studies have shown inconsistent association between dietary fiber intake and serum estrogen. Conjugated estrogens in the liver are excreted into the bile and reabsorbed in the intestine and fiber may bind estrogens in the colon during the enterohepatic circulation and increase the fecal excretion of estrogens [25-26]. Fiber may also act by reducing $\beta$-glucuronidase activity in the feces leading to a decrease in the reabsortion of estrogen in the colon [27]. Furthermore, high intake of dietary fiber may reduce breast cancer risk by reducing the risk of overweight and obesity. 
The second explanation is that beans contain a wide range of flavonoids and is the source of the greatest amount of flavonoids intake in the Nigerian diet. The Mexican diet is also rich in flavonoids, including flavones and lignans from beans, corn and green vegetables [28]. A hospital-based case-control study conducted in Mexico City found that in postmenopausal women, high dietary intake of flavonols and flavones was associated with a significant reduction of breast cancer risk [28]. Flavonoids may reduce carcinogenesis by preventing oxidative stress, inhibiting cell proliferation, inducing cell-cycle arrest, and maintaining DNA methylation [12, 29-31].

Lastly, women who have high beans' intake may also have retained other components of the traditional African diets, which are rich in anti-cancer agents. Whereas, women who reported never eating beans may have replaced it with other food items that are associated with increased risk for breast cancer such as charred red/organ meats, fat and calories[33]. However, more studies are needed to test these hypotheses because we did not examine them in the current study.

The limitations of our study include recall bias, and potential impact of breast cancer on dietary intake in general and specifically of bean intake. We did not adjust for family history of breast cancer, but previous studies showed low prevalence of this risk factor in Nigerian breast cancer patients. We also did not adjust for intake of other foods and nutrients. However, we adjusted for alcohol intake, the dietary factor that is most consistently associated with breast cancer risk as well as obesity measured by BMI and WHR. We used a modified FFQ, which has not been extensively validated in African populations. Despite these limitations, the strengths of our study include histological and immunohistochemical confirmation of breast cancer and its molecular subtypes, a large sample size with sufficient power to detect significant results, inclusion of a broad range of well-established covariates and confounders such as $\mathrm{BMI}$, menopausal status, demographic variables, types of occupation, as well as relative homogeneity of the study population.

\section{Conclusion}

We found a significant association between high intake of beans and reduced risks of total, hormone receptor-positive and triple-negative breast cancer in urbanized Nigerian women. In LMICs where the incidence of breast cancer is rising, increased dietary intake of bean can significantly reduce the incidence of breast cancer, particularly of the more aggressive subtype as triple-negative breast cancer, which is more prevalent in women in Sub-Saharan Africa. Future studies are needed to evaluate the causality of these relationships and the specific component of beans that might be attributed to preventing breast cancer in African women.

\section{Declarations}

\section{Ethical approval and consent to participate}


Ethical approval was obtained from the National Health Research Ethics Committee of Nigeria, Health Research Ethics Committees in each participating hospital and the institutional ethics committees at the University of Maryland School of Medicine, Baltimore (US). All participants gave written informed consent in accordance with the Declaration of Helsinki and the Nigerian National Code for Health Research Ethics.

\section{Availability of data and materials}

The datasets used and/or analyzed during the current study are available from the corresponding author on reasonable request.

\section{Conflict of Interest information}

The authors declare that they have no Conflict of Interest information

\section{Acknowledgments}

The project described was supported by the Training Program in Nigeria for Non-communicable Diseases Research (TRAPING NCD, D43TW009106) from the Fogarty International Centre; the African Female Breast Cancer Epidemiology (AFBRECANE, U01HG009784) and the African Collaborative Center for Microbiome and Genomics Research grants (ACCME U54HG006947) from the NIH Office of the Director/NHGRI. Additional support was received from the Maryland Department of Health's Cigarette Restitution Fund, the University of Maryland Greenebaum Comprehensive Cancer Center Support Grant (P30CA134274) and the American Cancer Society Institutional Research Grant IRG-18-160-16. The funding agencies did not play any role in data collection, analysis or publication. We acknowledge the staff of the Research Department of the Institute of Human Virology, Nigeria and clinical sites where participants were enrolled.

\section{Authors' Contributions}

CAA had the idea for the NIBBLE study, designed it, obtained funding, supervised implementation, and data management. SA contributed to the study design, data management, data analysis. GB conducted the statistical analysis and generated the first draft of the manuscript which was revised by the coauthors. TY, MY, OO, OB, EE, IS, EM, IA, and BA enrolled participants, conducted biopsies, data collection and ensured data quality. AF and BA conducted the laboratory diagnosis All authors contributed to drafting the manuscript, provided critical revisions and approved the final draft.

\section{References}

1. WHO, World Health Organization (accessed May 24 2021) The International Agency for Research on Cancer, press release 12 September 2018. https://www.who.int/cancer/PRGlobocanFinal.pdf?ua=1

2. Ingenbleek L, Jazet E, Dzossa AD, Adebayo SB, Ogungbangbe J, Dansou S et al (2017) Methodology design of the regional Sub-Saharan Africa Total Diet Study in Benin, Cameroon, Mali and Nigeria. Food Chem Toxicol 109(Pt 1):155-169 
3. Bray F, Ferlay J, Soerjomataram I, Siegel RL, Torre LA, Jemal A (2018) Global cancer statistics 2018: GLOBOCAN estimates of incidence and mortality worldwide for 36 cancers in 185 countries. $C A$ Cancer J Clin 68(6): 394-424

4. Adeloye D, Sowunmi OY, Jacobs W et al (2018) Estimating the incidence of breast cancer in Africa: a systematic review and meta-analysis. J Glob Health 8(1): 010419

5. Balekouzou A, Yin P, Pamatika CM et al (2017) Reproductive risk factors associated with breast cancer in women in Bangui: a case-control study. BMC Womens Health 17(1):14

6. Brinton LA, Figueroa JD, AwuahB et al (2014) Breast cancer in Sub-Saharan Africa: opportunities for prevention. Breast Cancer Res Treat 144(3): 467-478

7. Adebamowo CA, Ogundiran TO, Adenipekun AA et al (2003) Obesity and height in urban Nigerian women with breast cancer. Ann Epidemiol 13(6): 455-461

8. Akarolo-Anthony SN, Willett WC, SpiegelmanD, Adebamowo CA (2014) Obesity epidemic has emerged among Nigerians. BMC Public Health 14:455

9. Jacobs I, Taljaard-Krugell C, Ricci C et al (2019) Dietary intake and breast cancer risk in black South African women: the South African Breast Cancer study. Br J Nutr 121(5):591-600

10. Maiyoh GK, Tuei VC (2018) Rising Cancer Incidence and Role of the Evolving Diet in Kenya. Nutr Cancer 71(4):531-546

11. Katsidzira L, Gangaidzo I, Thomson S et al (2017) The shifting epidemiology of colorectal cancer in sub-Saharan Africa. Lancet Gastroenterol Hepatol 2(5):377-383

12. Adebamowo CA, Cho E, Sampson L, Katan MB, Spiegelman D, Willett WC, Holmes MD (2005) Dietary flavonols and flavonol-rich foods intake and the risk of breast cancer. Int J Cancer 114(4):628-633

13. Murtaugh MA, Sweeney C, Giuliano AR, Herrick JS et al (2008) Diet patterns and breast cancer risk in Hispanic and non-Hispanic white women: the Four-Corners Breast Cancer Study. Am J Clin Nutr 87(4):978-984

14. Sangaramoorthy M, Koo J, John EM (2018) Intake of bean fiber, beans, and grains and reduced risk of hormone receptor-negative breast cancer: the San Francisco Bay Area Breast Cancer Study. Cancer Med 7(5):2131-2144

15. Velie EM, Schairer C, Flood A, He JP, Khattree R, Schatzkin A (2005) Empirically derived dietary patterns and risk of postmenopausal breast cancer in a large prospective cohort study. Am J Clin Nutr 82(6):1308-1319

16. Jedy-Agba E, McCormack V, Olaomi O, Badejo W, Yilkudi M et al (2017) Determinants of stage at diagnosis of breast cancer in Nigerian women: sociodemographic, breast cancer awareness, health care access and clinical factors. Cancer Causes Control 28(7):685-697

17. Christgen M, Langer F, Kreipe H (2016) Histological grading of breast cancer. Pathologe 37(4):328336

18. Hammond ME, Hayes DF, Dowsett M, Allred DC et al (2010) Pathologists' Guideline Recommendations for Immunohistochemical Testing of Estrogen and Progesterone Receptors in 
Breast Cancer. Breast Care (Basel) 5(3):185-187

19. Wolff AC, Hammond ME, Schwartz JN et al (2007) American Society of Clinical Oncology/College of American, P. American Society of Clinical Oncology/College of American Pathologists guideline recommendations for human epidermal growth factor receptor 2 testing in breast cancer. Arch Pathol Lab Med 131(1):18-43

20. Waks AG, Winer EP (2018) Breast Cancer Treatment: A Review. JAMA 321(3):288-300

21. Adebamowo CA, Ogundiran TO, Adenipekun AA et al (2003) Waist-hip ratio and breast cancer risk in urbanized Nigerian women. Breast Cancer Res 5(2):R18-R24

22. Filmer D, Pritchett LH (2001) Estimating wealth effects without expenditure data-or tears: an application to educational enrollments in states of India. Demography 38(1):115-132

23. Thompson MD, Mensack MM, Jiang W, Zhu Z, Lewis MR, McGinley JN, Thompson HJ (2012) Cell signaling pathways associated with a reduction in mammary cancer burden by dietary common bean (Phaseolus vulgaris L.). Carcinogenesis 33(1):226-232. doi:10.1093/carcin/bgr247

24. Cruz-Bravo RK, Guevara-Gonzalez RG, Ramos-Gomez M, Oomah BD, Wiersma P, Campos-Vega R, Loarca-Pina G (2014) The fermented non-digestible fraction of common bean (Phaseolus vulgaris L.) triggers cell cycle arrest and apoptosis in human colon adenocarcinoma cells. Genes Nutr 9(1):359. doi:10.1007/s12263-013-0359-1

25. Aune D, Chan DS, Greenwood DC, Vieira AR, Rosenblatt DA, Vieira R, Norat T (2012) Dietary fiber and breast cancer risk: a systematic review and meta-analysis of prospective studies. Ann Oncol 23(6):1394-1402. doi:10.1093/annonc/mdr589

26. Farvid MS, Spence ND, Holmes MD, Barnett JB (2020) Fiber consumption and breast cancer incidence: A systematic review and meta-analysis of prospective studies. Cancer. doi:10.1002/cncr.32816

27. Gaskins AJ, Mumford SL, Zhang C, Wactawski-Wende J, Hovey KM, Whitcomb BW, Study G (2009) Effect of daily fiber intake on reproductive function: the BioCycle Study. Am J Clin Nutr, 90(4), 10611069. doi:10.3945/ajcn.2009.27990

28. Torres-Sanchez L, Galvan-Portillo M, Wolff MS, Lopez-Carrillo L (2009) Dietary consumption of phytochemicals and breast cancer risk in Mexican women. Public Health Nutr 12(6):825-831. doi:10.1017/S136898000800325X

29. Carlos-Reyes A, Lopez-Gonzalez JS, Meneses-Flores M, Gallardo-Rincon D, Ruiz-Garcia E, Marchat LA, Lopez-Camarillo C (2019) Dietary Compounds as Epigenetic Modulating Agents in Cancer. Front Genet 10:79. doi:10.3389/fgene.2019.00079

30. Feng XL, Ho SC, Mo XF, Lin FY, Zhang NQ, Luo H, Zhang (2019) C. X. Association between flavonoids, flavonoid subclasses intake and breast cancer risk: a case-control study in China. Eur J Cancer Prev. doi:10.1097/CEJ.0000000000000561

31. Hui C, Qi X, Qianyong Z, Xiaoli P, Jundong Z, Mantian M (2013) Flavonoids, flavonoid subclasses and breast cancer risk: a meta-analysis of epidemiologic studies. PLoS ONE 8(1):e54318. doi:10.1371/journal.pone.0054318 
32. Mourouti N, Kontogianni MD, Papavagelis C, Panagiotakos DB (2015) Diet and breast cancer: a systematic review. Int J Food Sci Nutr 66(1):1-42. doi:10.3109/09637486.2014.950207

33. Keding GB, Msuya JM, Maass BL, Krawinkel MB (2011) Dietary patterns and nutritional health of women: the nutrition transition in rural Tanzania. Food Nutr Bull 32(3):218-226. doi: $10.1177 / 156482651103200306$

\section{Tables}

Table 1 Descriptive characteristics of the study sample, the Nigerian Integrative Epidemiology of Breast Cancer Study (NIBBLE), year 2014-2016. 
$\mathrm{N}=944$

Cases ${ }^{\text {a }} \mathrm{N}=472 \quad$ Control ${ }^{\text {a }} \mathrm{N}=472 \quad p$-value ${ }^{a}$

$N(\%) /$ Mean $\pm S D \quad N(\%) /$ Mean $\pm S D$

Age, years

$44.4 \pm 10.0$

$43.5 \pm 9.5$

$0.18^{+}$

Age at first menstrual period, years

$14.4 \pm 1.7$

$14.5 \pm 1.9$

0.23

$\mathrm{BMI} \mathrm{kg} / \mathrm{m}^{2}$

0.01

\begin{tabular}{|c|c|c|c|c|}
\hline & $<25$ & $147(31.1)$ & $116(24.6)$ & \\
\hline & $25-29.99$ & $162(34.3)$ & $161(34.1)$ & \\
\hline & $\geq 30$ & $147(31.2)$ & $185(39.2)$ & \\
\hline & Missing & $16(3.4)$ & $10(2.1)$ & \\
\hline WHR & & & & 0.07 \\
\hline & High, $>0.85$ & $330(69.9)$ & $306(64.8)$ & \\
\hline & Missing & $16(3.4)$ & $14(3.0)$ & \\
\hline Education & & & & 0.01 \\
\hline & Elementary $\leq$ & $135(28.6)$ & 94 (19.9) & \\
\hline & Complete HS & $101(21.4)$ & $120(25.4)$ & \\
\hline & Post HS no university & $118(25.0)$ & $111(23.6)$ & \\
\hline & Completed University & $112(23.7)$ & $145(30.7)$ & \\
\hline & Missing & $6(1.3)$ & $2(0.4)$ & \\
\hline Marital status & & & & 0.93 \\
\hline & Married & $341(72.3)$ & $344(72.9)$ & \\
\hline & Single & $53(11.2)$ & $54(11.4)$ & \\
\hline & Separated/Divorced/Widowed & $72(15.3)$ & $73(15.5)$ & \\
\hline & Missing & $6(1.3)$ & $1(0.2)$ & \\
\hline Occupation & & & & $<0.01$ \\
\hline & Self-employed & $124(26.3)$ & $49(10.4)$ & \\
\hline & Unskilled manual & $197(41.7)$ & $258(54.7)$ & \\
\hline & Skilled manual & $112(23.7)$ & $76(16.1)$ & \\
\hline & Professional/executive & $22(4.7)$ & $71(15.0)$ & \\
\hline
\end{tabular}




\begin{tabular}{|c|c|c|c|}
\hline Missing & $17(3.6)$ & $18(3.8)$ & \\
\hline Wealth index & & & 0.43 \\
\hline Low & $183(38.8)$ & $188(39.8)$ & \\
\hline Middle & $152(32.2)$ & $123(26.1)$ & \\
\hline High & $126(26.7)$ & $154(32.6)$ & \\
\hline Missing & $11(2.3)$ & $7(1.5)$ & \\
\hline Ever used oral contraceptives & & & 0.25 \\
\hline Yes & $141(29.9)$ & $160(33.9)$ & \\
\hline Missing & $11(2.3)$ & $6(1.3)$ & \\
\hline Number of pregnancies & & & 0.97 \\
\hline 0 & $49(10.4)$ & $45(9.5)$ & \\
\hline $1-2$ & $86(18.2)$ & $95(20.1)$ & \\
\hline $3-5$ & $227(48.1)$ & $231(48.9)$ & \\
\hline$\geq 6$ & $99(21.0)$ & $98(20.9)$ & \\
\hline Missing & $11(2.3)$ & $3(0.6)$ & \\
\hline Menopausal status & & & 0.05 \\
\hline Postmenopausal & $167(35.4)$ & $146(30.9)$ & \\
\hline Missing & $6(1.3)$ & $2(0.4)$ & \\
\hline Ever breastfed more than one month & & & 0.21 \\
\hline Yes & $364(77.1)$ & 377 (79.9) & \\
\hline Missing & $14(3.0)$ & $14(3.0)$ & \\
\hline Smoke & & & NA \\
\hline Yes & $2(0.4)$ & $3(0.6)$ & \\
\hline Missing & $7(1.5)$ & $2(0.4)$ & \\
\hline Alcohol use & & & 0.06 \\
\hline Yes & $100(21.2)$ & $79(16.7)$ & \\
\hline Missing & $9(1.9)$ & $4(0.9)$ & \\
\hline Leisure-time physical activity (meet th & nmendations) & & $<0.01$ \\
\hline
\end{tabular}




\begin{tabular}{llll}
\hline & Physical active & $83(17.6)$ & $135(28.6)$ \\
\hline & Physical inactive & $356(75.4)$ & $322(68.2)$ \\
\hline Beans intake & Missing & $33(7.0)$ & $15(3.2)$ \\
\hline & Never (in the past year) & $241(51.1)$ & $185(39.2)$ \\
\hline & Low ( $\leq 1$ portion/week) & $154(32.6)$ & $186(39.4)$ \\
\hline & High (>1 portion/week) & $77(16.3)$ & $101(21.4)$ \\
\hline${ }^{+}$Cases and controls are matched by age ( \pm 5 years) & & \\
BMI, Body Mass Index; WHR, Waist-Hip Ratio; HS, High School; WHO, World Health Organization
\end{tabular}

Table 2. Age-adjusted and multivariable models of total beans intakes per week and risk of breast cancer in the Nigerian Integrative Epidemiology of Breast Cancer Study (NIBBLE), the year 2014-2016

\begin{tabular}{lllll} 
& \multicolumn{2}{l}{ Age-adjusted model $^{\mathrm{a}}$} & \multicolumn{2}{l}{ Multivariable model $^{\mathrm{a} \mathrm{b}}$} \\
\cline { 2 - 5 } & OR $(95 \% \mathrm{Cl})$ & P-value & ${ }^{\mathrm{b}}$ OR $(95 \% \mathrm{Cl})$ & P-value \\
\hline Beans intake & & $<0.01^{\mathrm{c}}$ & & $<0.01^{\mathrm{c}}$ \\
\hline Never (in the past year) & 1 & & 1 & 1 \\
\hline Low ( $\leq 1$ portion/week) & $0.63(0.47-0.84)$ & $<0.01$ & $0.60(0.43-0.84)$ & $<0.01$ \\
\hline High (>1 portion/week) & $0.59(0.41-0.84)$ & $<0.01$ & $0.57(0.38-0.85)$ & $<0.01$
\end{tabular}

${ }^{\text {a }}$ Cases and controls were matched by age ( \pm 5 years) analyses were done using a conditional logistic regression model.

${ }^{b}$ The models were adjusted for age, WHR, BMI, occupation, menopause status, alcohol use, and physical activity.

${ }^{\mathrm{C}} \mathrm{P}$-value for trend

OR, Odds Ratio; Cl, Confidence Interval;

Table 3 Age-adjusted and multivariable models of total beans intakes per week and risk of breast cancer subtypes in the Nigerian Integrative Epidemiology of Breast Cancer Study (NIBBLE), the year 2014-2016. 
HR (ER+/PR+)/her2-

Age-Adjusted

model $^{a}$

OR

(95\% Cl)
Multivariable

Model $^{\text {a b }}$

OR

(95\% Cl)
TNBC

Age-

Multivariable

Adjusted model ${ }^{a}$ Model $^{\mathrm{a}} \mathrm{b}$

OR

OR

$(95 \% \mathrm{Cl})$

(95\% Cl)

Beans intake

Never (in the past $\quad 1.00$ year)

Low $(\leq 1$

portion/week)

0.73

0.79

0.67

0.70

(0.45-1.18)

(0.47-1.33)

(0.44-1.02)

$(0.43-1.12)$

High ( $>1$

portion/week)

0.45

0.45

0.41

0.47

(0.24-0.86)

(0.23-0.90)

(0.23-0.73)

(0.25-0.88)

$P$-value for trend

0.01

0.02

$<0.01$

0.01

a Unconditional logistic regression models with complete study sample

b The models were adjusted for age, WHR, BMI, occupation, menopause status, alcohol use, and physical activity.

HP, Hormone Receptor Positive; ER, estrogen; PR, progesterone; her2, human epidermal growth factor-2; TNBC, Triple-Negative Breast Cancer; OR, Odds Ratio; Cl, Confidence Interval

\section{Figures}
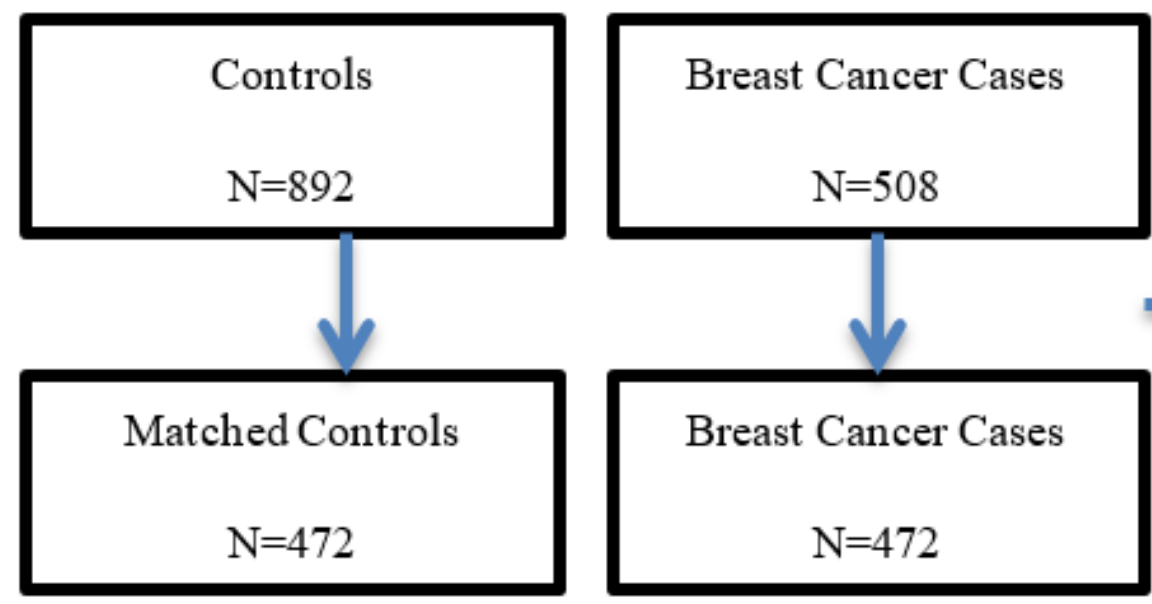

Excluded:

Unmatched controls $=420$

Unmatched breast cancer

$$
\text { cases }=36
$$

Figure 1 
$<p>$ Flowchart describing the final matched case control sample for the overall breast cancer analysis $</ p>\langle p\rangle\langle/ p\rangle\langle p\rangle\langle/ p\rangle$

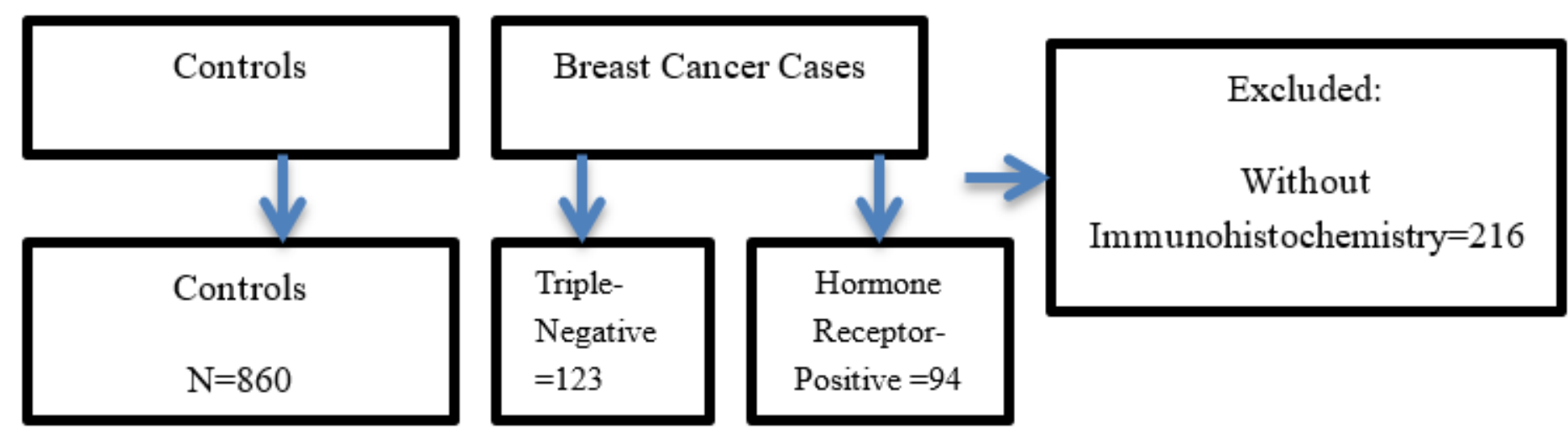

Figure 2

$\langle p>$ Flowchart describing the final subsamples for the breast cancer subtype analyses\&nbsp; $</ p>$ 